\title{
Laser-Driven Anharmonic Oscillator: Ground-State Dissociation of the Helium Hydride Molecular Ion by Midinfrared Pulses
}

\author{
Philipp Wustelt, ${ }^{1,2, *}$ Florian Oppermann $\odot{ }^{3}$ Saurabh Mhatre $\odot,{ }^{4}$ Matthias Kübel $\odot,{ }^{1,2}$ \\ A. Max Sayler, ${ }^{1,2, \$}$ Manfred Lein, ${ }^{3}$ Stefanie Gräfe, ${ }^{4}$ and Gerhard G. Paulus $\circledast^{1,2, \dagger}$ \\ ${ }^{1}$ Institute of Optics and Quantum Electronics, Friedrich Schiller University Jena, D-07743 Jena, Germany \\ ${ }^{2}$ Helmholtz. Institute Jena, Fröbelstieg 3, 07743 Jena, Germany \\ ${ }^{3}$ Institut für Theoretische Physik, Leibniz Universität Hannover, Appelstraße 2, 30167 Hannover, Germany \\ ${ }^{4}$ Institute of Physical Chemistry, Friedrich Schiller University Jena, Helmholtzweg 4, 07743 Jena, Germany
}

(Received 5 January 2021; revised 6 April 2021; accepted 27 April 2021; published 20 July 2021)

\begin{abstract}
The vibrational motion of molecules represents a fundamental example of an anharmonic oscillator. Using a prototype molecular system, $\mathrm{HeH}^{+}$, we demonstrate that appropriate laser pulses make it possible to drive the nuclear motion in the anharmonic potential of the electronic ground state, increasing its energy above the potential barrier and facilitating dissociation by purely vibrational excitation. We find excellent agreement between the frequency-dependent response of the helium hydride molecular cation to both classical and quantum mechanical simulations, thus removing any ambiguities through electronic excitation. Our results provide access to the rich dynamics of anharmonic quantum oscillator systems and pave the way to stateselective control schemes in ground-state chemistry by the adequate choice of the laser parameters.
\end{abstract}

DOI: 10.1103/PhysRevLett.127.043202

The model system of an oscillator in a harmonic potential is of fundamental importance for a plethora of phenomena in nature. The assumption that the restoring force is linear to the displacement is, however, only a firstorder approximation. Usually it is not fulfilled, and the complex dynamics in anharmonic potentials must be considered. A very prominent example is the description of molecular bonds [1,2]. As for any oscillator, the anharmonicity becomes increasingly important for increasing oscillation amplitudes and is of particular relevance for transformation or dissociation of the molecule.

Intense laser pulses provide an efficient means to control dissociation of molecules [3]. If the light couples directly to the vibrational degrees of freedom of the molecule, absorption of one or more photons can excite IR-active transitions, transferring population from lower to higher vibrational bound or even continuum states while remaining on the same electronic state [4-6]. This process can drive nuclear motion to distances far from equilibrium in the anharmonic potential of the electronic ground state and even lead to bond breakage.

In diatomic molecules without a permanent dipole moment, as is the case for the symmetric $\mathrm{H}_{2}^{+}$[7], direct vibrational excitation is not possible. In that case,

Published by the American Physical Society under the terms of the Creative Commons Attribution 4.0 International license. Further distribution of this work must maintain attribution to the author(s) and the published article's title, journal citation, and DOI. dissociation can only proceed by coupling to higher-lying electronic states. Bond breaking by the laser-driven electron motion constitutes the usual pathway of photochemical reactions in intense laser fields and has been extensively studied $[4,7,8]$.

While molecular dissociation driven exclusively by permanent dipole excitations has been studied theoretically for small molecules [5,6,9-11], instances of its experimental observation have remained scarce. This is remarkable since the ground electronic surface is of enormous general importance for chemical syntheses, which are predominantly carried out on the ground state [12-15]. The major challenges for the experimental observation of ground-state dissociation are the timescale and the anharmonicity of molecular vibrations [16].

Efficient population transfer between two levels is only possible in the case of resonance. The resonances for transitions between vibrational levels are typically located in the midinfrared spectral range. This contrasts with a large body of experiments that have efficiently driven electronic dynamics using intense visible or near-infrared light [4]. Since electronic excitations tend to dominate the molecular response in strong laser fields and lead to a fast dephasing of vibrational dynamics, the observation of direct vibrational excitation is obscured.

Moreover, the direct excitation from the vibrational ground state to an unbound vibrational state within the manifold of the electronic ground state is extremely unlikely (see the Supplemental Material [17]). The required intensity would be beyond the onset of ionization [18]. Therefore, for the unambiguous observation of ground-state dissociation, 


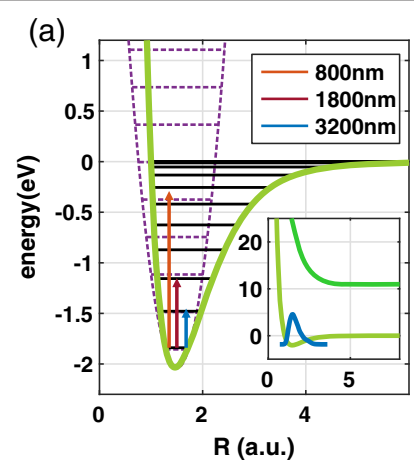

(b)

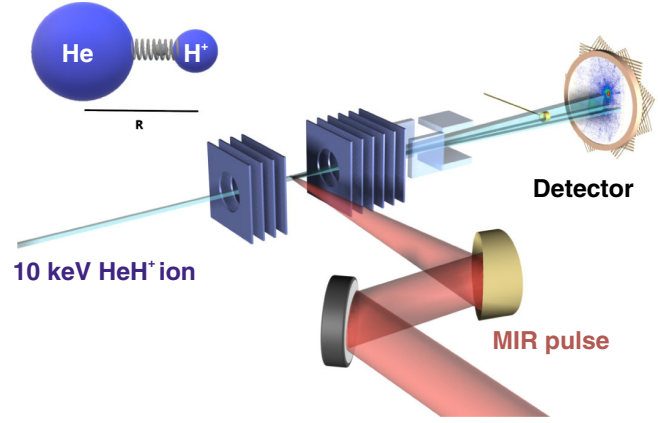

(c)

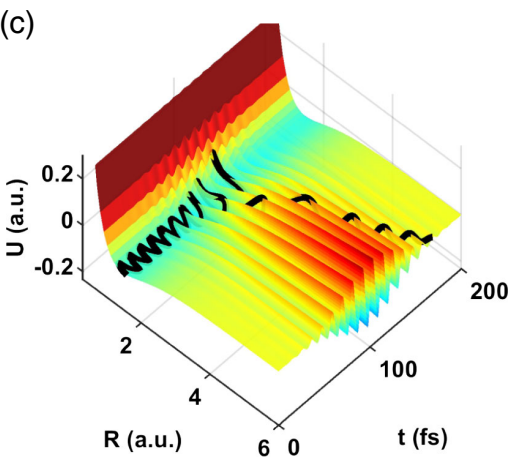

FIG. 1. Laser-driven anharmonic oscillator. (a) The ground state $\left(X^{1} \Sigma^{+}\right)$of $\mathrm{HeH}^{+}$(solid green) and the vibrational levels of the anharmonic potential of $\mathrm{HeH}^{+}$are compared to a harmonic potential (dashed purple). Midinfrared (MIR) photons can couple these states as consequence of the permanent dipole moment of $\mathrm{HeH}^{+}$. The inset enlarges and displays the potential energy curves for the two electronic ground states $\left(X^{1} \Sigma^{+}\right)$and the first excited state $\left(A^{1} \Sigma^{+}\right)$. The large separation makes electronic excitation very unlikely. Panel

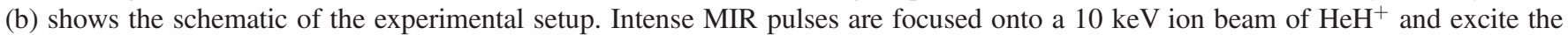
molecule up to dissociation in the electronic ground state. The ionic and neutral fragments are detected in coincidence by a delay line detector. Weak electrostatic fields allow for separation of different fragments. (c) Illustration of a classical trajectory model for laserinduced dissociation of $\mathrm{HeH}^{+}$. Shown are the effective potential and the resulting motion on the $X^{1} \Sigma^{+}$surface for a laser field with $3.2 \mu \mathrm{m}$ and $50 \mathrm{fs}$ at a peak intensity of $30 \mathrm{TW} / \mathrm{cm}^{2}$.

the excitation of intermediate vibrational states using midinfrared (MIR) light is necessary ("vibrational ladder climbing"). However, as consequence of the anharmonic potential [see Fig. 1(a)], consecutive vibrational transitions require different frequencies for resonance.

The uneven spacing of vibrational states results in a characteristic wavelength dependence of the dissociation probability $[5,6,19]$. It has been predicted that chirped laser pulses increase dissociation [20-22]. Such vibrational ladder climbing has been studied [23-25] and dissociation induced by MIR femtosecond has been indirectly measured in coordination complexes of metal carbonyls using infrared spectroscopy $[12,26,27]$.

Here, we present direct measurements of molecular photodissociation induced by purely vibrational excitation. The absolute yield as well as the features in the measured momentum distribution of the fragments allow for an unambiguous determination of the pathways of the molecular breakup and a detailed comparison with theoretical modeling. We show that the laser-driven dynamics can be adequately described by the intuitive model of a driven anharmonic oscillator. We choose the simplest heteronuclear molecule, the $\mathrm{HeH}^{+}$-molecular ion. $\mathrm{HeH}^{+}$possesses a number of favorable properties: its large asymmetry results in a strong permanent dipole moment, while the electronic ground state $X^{1} \Sigma^{+}$and first excited state $A^{1} \Sigma^{+}$are spaced sufficiently far apart [see inset of Fig. 1(a)] to make electronic excitation irrelevant for the laser parameters used here [28-31].

As predicted by the driven anharmonic oscillator model, a prerequisite for efficient dissociation is the match of the periodicity of the driving force to the eigenfrequency of the system, which is for $\mathrm{HeH}^{+} 11.5$ fs. This is realized by employing MIR laser pulses, which activates the direct dissociation in the electronic ground state. The experiment is conducted combining a molecular ion beam target for coincidence 3D momentum spectroscopy with an optical parametric amplifier. Laser pulses with preselected wavelength were focused onto a beam of $\mathrm{HeH}^{+}$ions, which were formed in a duoplasmatron ion source [see Fig. 1(b)] [32]. The full 3D momenta of the laser-induced fragments are measured in coincidence and as a function of the wavelength (see the Supplemental Material [17] for details).

To characterize the frequency-dependent response, we varied the periodicity of the driving laser in the range between 1.3 and 13 fs [see Fig. 2(a)]. Considering the applied laser intensities shows that the dissociation yield increases for increasing periods of the laser field. Given the spacing of vibrational states shown in Fig. 1(a), this is a clear indication that purely vibrational excitation rather than electronic excitation is responsible for the dissociation of $\mathrm{HeH}^{+}$. Additional confirmation is that the channel $\mathrm{HeH}^{+}+n \hbar \omega \rightarrow \mathrm{He}^{+}+\mathrm{H}$, which would require electronic excitation, was not observed for any wavelength.

The frequency dependence can be understood using two different approaches. An intuitive picture of the nuclear motion is provided by the classical description of a driven oscillator. In this picture, a classical particle with the reduced mass of $\mathrm{HeH}^{+}$is placed in the ground-state potential surface displayed in Fig. 1(a). For small oscillation amplitudes, the field-free motion is well described by a sinusoidal motion with a period of $11.5 \mathrm{fs}$, as can be seen in Fig. 1(c), where the classical model is illustrated. When the laser field is switched on, it provides an external force that drives the vibrating motion of the nuclei. If the laser field oscillates with a similar periodicity as the nuclear motion, the system is resonantly excited and the oscillation amplitude rises quickly. For increasing elongations from the equilibrium 
distance, the molecular potential deviates from a harmonic potential. Even if the sinusoidal oscillation of the driving laser field deviates from the anharmonic motion of the nuclei, the external force can eventually separate the nuclei so far from each other that the oscillator is destroyed.

A more rigorous picture is given by a quantum description. The absorption of photons can transfer population between low and higher bound and even unbound vibrational states. In principle, absorption of a single photon with sufficient energy $(\lambda<671 \mathrm{~nm})$ to overcome the nuclear binding potential could lead to dissociation. However, transitions from higher bound states to the continuum are much more probable than those coming from low bound states (see the Supplemental Material [17]). Thus, efficient dissociation from $v=0$ requires the excitation of higher bound states before continuum states are efficiently populated [29]. Depending on the photon energy, different pathways toward the continuum are possible. The anharmonicity can lead to complex dynamics such as overtone absorption, nonresonant transitions, or tunneling, i.e., in the language of vibrational ladder climbing, the molecule does not climb all the rungs of the ladder one after the other but, depending on the laser frequency, leaves some rungs out on the way up.

The measured dissociation yields [see Fig. 2(a)] agree well with results obtained from both classical and quantum dynamical simulations. The laser-induced excitation on the electronic ground state is computed, first by solving the classical motion for an ensemble of classical particles on the corresponding potentials and, in the second case, by solving the time-dependent Schrödinger equation (see the Supplemental Material [17] for details). Further, comparing the quantum and the classical results demonstrates that the classical model is similarly appropriate to predict the dynamics. The general trend agrees with earlier theoretical results for other molecules, e.g., HF $[6,19]$. It was predicted that due to the anharmonicity of the potential, a laser oscillation period of $T_{L} \approx 1.1-1.2 T_{01}$, which is slightly longer than the ground vibrational period $T_{01}=11.5 \mathrm{fs}$, maximizes the photodissociation probability. In good agreement, our simulations indicate a maximum at $T_{L} \approx 13$ fs [see Fig. 2(b)]. For larger $T_{L}$, i.e., smaller photon energies, the transfer from $v=0$ to higher states becomes increasingly difficult. Thus, the dissociation probability is reduced for $T_{L} \gg T_{01}$. For such long periods, the contributions from vibrational states $v>0$, which are populated in the high-temperature ion source [33], become highly relevant [see blue curves in Fig. 2(b)]. Without the vibrational averaging, i.e., considering only $v=0$, the wavelength dependence is significantly narrower, but the position of the maximum is unchanged [see green curves in Fig. 2(b)].

In addition to the dissociation probability, we examined the measured momenta of the fragments after dissociation for $\mathrm{HeH}^{+}$and the kinetic energy release (KER). For
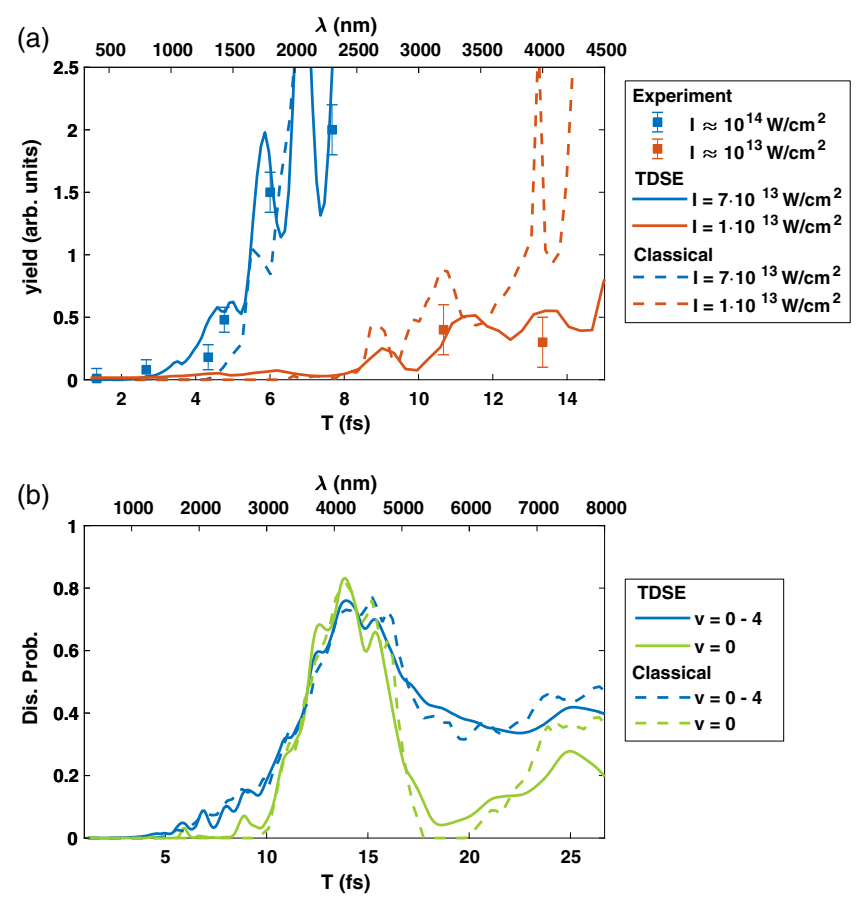

FIG. 2. Frequency-dependent response of $\mathrm{HeH}^{+}$. (a) Yield for $\mathrm{HeH}^{+}+n \hbar \omega \rightarrow \mathrm{He}+\mathrm{H}^{+}$as function of the periodicity of the driving field and laser wavelength (upper abscissa). Measured results are displayed together with one-dimensional timedependent Schrödinger equation (TDSE) and classical simulations. The computational results have been averaged over a distribution of initial vibrational states $(v=0-4)$ according to the temperature of the $\mathrm{HeH}^{+}$source. To account for the lower laser intensity at long wavelengths, simulations have been performed for two intensity values $\left(1 \times 10^{13} \mathrm{~W} / \mathrm{cm}^{2}\right.$ and $\left.7 \times 10^{13} \mathrm{~W} / \mathrm{cm}^{2}\right)$. For better clarity, in the simulation a constant pulse duration of 50 fs was used, although the pulse duration in the experiment varied by a factor of 2 due to technical reasons (see the Supplemental Material [17] for details). Note that longer pulse durations can result in higher dissociation yields, but the assumption of a wavelength-independent pulse duration does not influence the interpretation of this work. In (b), for a larger frequency range, the theoretical dissociation probability of the classical and the quantum mechanical simulations is compared at an intensity of $7 \times 10^{13} \mathrm{~W} / \mathrm{cm}^{2}$ for the vibrational ground state ( $v=0$ ) and vibrationally averaged over the states $v=0-4$. The second maximum around $T_{L} \approx 25 \mathrm{fs}$ is attributed to two-photon excitation of the $v=0 \rightarrow 1$ transition.

$T_{L}=6 \mathrm{fs}$, the KER spectrum reaches up to $\approx 1 \mathrm{eV}$ and reveals distinct peaks for certain KER values [see Figs. 3(a) and 3(b)]. The measured spectrum is dominated by a peak at $0.26 \mathrm{eV}$. Additional peaks at $0.1 \mathrm{eV}$ and $0.05 \mathrm{eV}$ are visible.

The KER position after absorption of $n$ photons can be estimated from the photon energy and the vibrational eigenenergies $E_{v}$ via KER $=n \hbar \omega_{L}+E_{v}-E_{D}$, with $E_{D}$ being the dissociation energy (see the Supplemental Material [17]). Several pathways starting from different $v$ and absorbing different $n$ photons are possible. To assess 


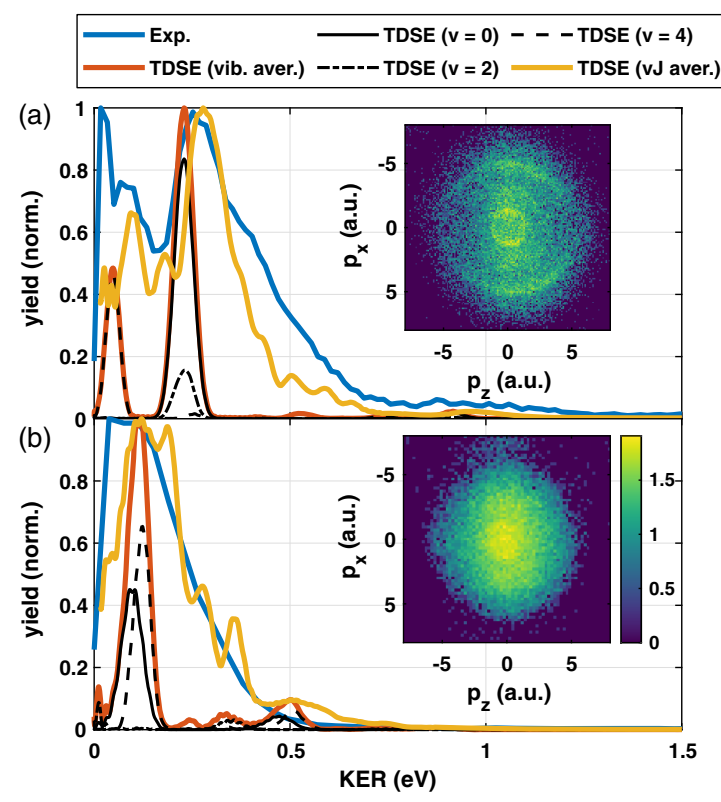

FIG. 3. Above-threshold dissociation of $\mathrm{HeH}^{+}$. (a) Measured kinetic energy release (KER) dependent dissociation yield (blue) for $\mathrm{T}_{L}=6 \mathrm{fs}(\lambda=1800 \mathrm{~nm})$ together with the vibrational and focal-volume-averaged 1D TDSE simulation (red) $\left(\mathrm{I} \approx 10 \times 10^{13} \mathrm{~W} / \mathrm{cm}^{2}\right.$ in the measurement and $\mathrm{I}_{0}=$ $7 \times 10^{13} \mathrm{~W} / \mathrm{cm}^{2}$ in the simulation). Along with the $v$-averaged distribution, the contributions of the initial states $v=0, v=2$, and $v=4$ are shown (black). Good agreement between measurement and simulation is obtained when averaging over vibrational and rotational states is included (yellow). The inset displays the measured momentum distribution in the polarization plane. (b) same for $T_{L}=10.7 \mathrm{fs}\left(\lambda_{L}=3200 \mathrm{~nm}\right.$, $\mathrm{I} \approx 1 \times 10^{13} \mathrm{~W} / \mathrm{cm}^{2}$ in the measurement and $\mathrm{I}_{0}=1.5 \times$ $10^{13} \mathrm{~W} / \mathrm{cm}^{2}$ in the simulation).

the actual dominating pathways, we compare our results to simulated KER spectra. The main peak at $\approx 0.22 \mathrm{eV}$ in the simulated spectrum originates from the vibrational ground state $v=0$ as well as $v=2$. This can be understood by inspecting the time-dependent evolution of the excitation dynamics in the quantum simulation. In Fig. 4, the timedependent population of vibrational states is plotted for various initial states. First, the population from $v=0$ is transferred to $v=2$ by one-photon absorption [cf. Fig. 1(a)]. From $v=2$, the population can be dissociated by two-photon absorption to a KER of $0.22 \mathrm{eV}$, or it is transferred by absorption of another photon to $v=5$. From this state, single-photon dissociation produces a KER of $0.25 \mathrm{eV}$. Both pathways lead to a KER close to the major feature at $\approx 0.26 \mathrm{eV}$ in the measured spectrum. Two-photon absorption starting at $v=5$ leads to a KER of $0.93 \mathrm{eV}$, visible as a small feature in the simulated spectrum. For the present laser period $T_{L}=6 \mathrm{fs}$, the initial $v=1$ and $v=3$ contribute only weakly to the dissociation yield. The state $v=4$ is the lowest state, which allows for direct transition to unbound states by absorption of a single photon. Thus,
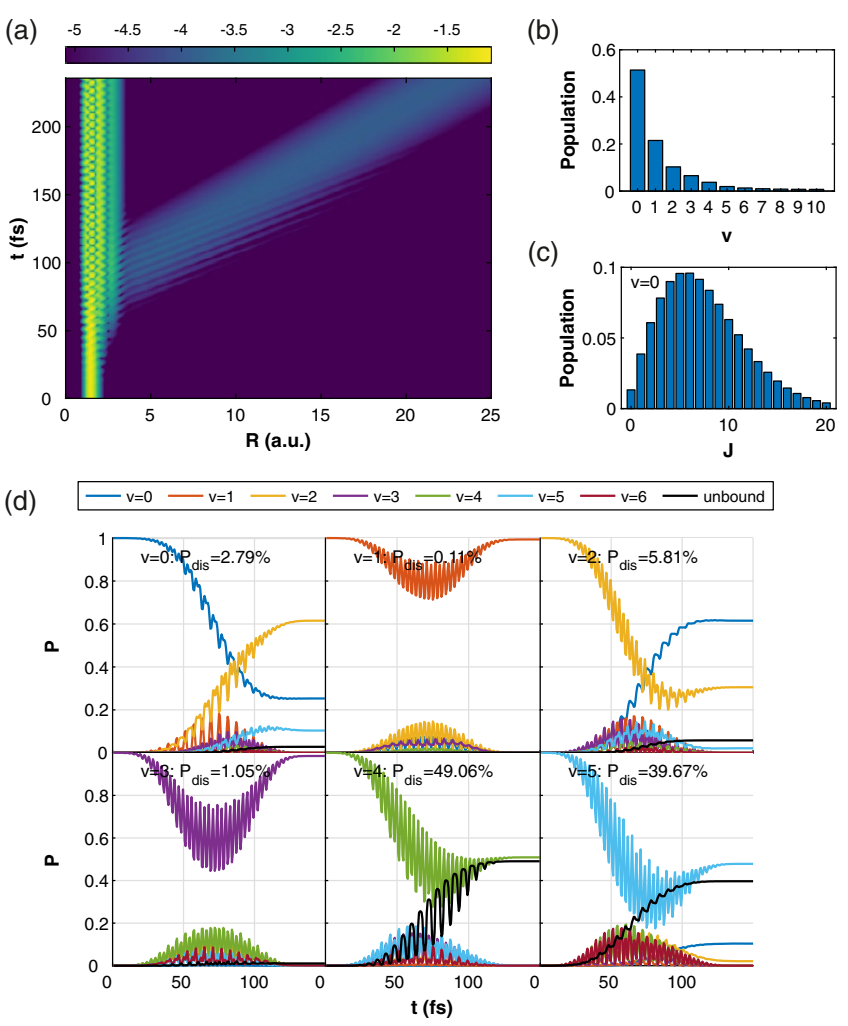

FIG. 4. Time dependence of the excitation dynamics. (a) Simulated evolution of a wave packet with $v=0$ and $J=0$ on the $X^{1} \Sigma^{+}$in a $1.8 \mu \mathrm{m}$ laser pulse with $50 \mathrm{fs}$ and a peak intensity of $\mathrm{I}=7 \times 10^{13} \mathrm{~W} / \mathrm{cm}^{2}$. Displayed is the time-dependent probability density (log scale). (b) shows the initial distribution of different $v$ used in the simulations. In addition, the population of rotational states, which is displayed in (c) for a temperature of $3100 \mathrm{~K}$, is influencing the KER spectrum. In (d), the detailed excitation pathways of vibrational states during the interaction of $\mathrm{HeH}^{+}$for different initial $v$ are shown. $P_{\text {dis }}$ denotes the final dissociation probability for each vibrational state. The major contribution to dissociation comes from $v=0, v=2$, and $v=4$. During the interaction, the excitation of the states $v>6$ remains low and is therefore not displayed.

the initial population from $v=4$ can efficiently be dissociated, resulting in a KER of $\approx 0.05 \mathrm{eV}$.

An analogous analysis can be performed for a different excitation periodicity at $T_{L}=10.7 \mathrm{fs}$ [see Fig. 3(b)]. Because of the smaller photon energy, the observed KER is shifted to smaller values. Furthermore, this photon energy is very close to the fundamental excitation $v=0 \rightarrow v=1$, so that the latter state is efficiently populated [see Fig. 1(a)]. From $v=1$, however, excitation to a higher state does not meet a resonance condition. Only a small part of the population is transferred to the states $v=2$ and $v=6$ and subsequently to dissociation. The state $v=4$ has a relatively high dissociation probability. The reason is that, by absorption of a single photon, the population transfer from $v=4$ to $v=6$ is close to resonance. The state $v=6$ is the lowest state that allows 
for one-photon dissociation, resulting in a KER of $\approx 0.12 \mathrm{eV}$.

A pronounced difference between measured and simulated spectra is the observation that the simulated spectra consist of several clearly separated peaks, while the measured spectra are much smoother. This can be explained by contributions of high rotational states, which are populated in the $\mathrm{HeH}^{+}$due to the high temperature of the ion source [see Fig. 4(c)] $[33,34]$. Clearly, only the combination of vibrational and rotational averaging yields a very good agreement with the measured curve [see Fig. 3(a)].

In conclusion, we have studied experimentally and computationally the multiphoton dissociation of $\mathrm{HeH}^{+}$ on the electronic ground state. The frequency-dependent response of the molecular system agrees very well with both classical and quantum mechanical simulations, demonstrating that our experiment represents the realization of a laser-driven anharmonic oscillator. While in a fast oscillating laser field the dominating fragmentation pathway is ionization and almost no dissociation can be observed, lower laser frequencies result in a strong increase of the dissociation probability.

The agreement of the experimental results with both classical and quantum simulations demonstrates that the results can be interpreted applying the anharmonic oscillator analogies. Although $\mathrm{HeH}^{+}$is a simple system, our work gives valuable new insight in the rich dynamics and the correspondence between quantum and classical mechanics for fundamental quantum systems.

Furthermore, our result represents an important step for controlled ground-state chemistry. We provide a benchmark for the selective bond cleavage via vibrational excitation, which is the key to active control over molecular reactions [14]. The results pave the route toward coherent control of Morse systems in intense fields and ultrafast state-selective preparation of molecules by tailored MIR pulses [35-37], which can provide efficient ways to study and manipulate various molecular phenomena such as the roaming of highly excited molecules [38]. We expect these concepts to become more and more important for the femtochemistry of more complex molecules with the increasing advance of midinfrared ultrashort laser sources.

The authors acknowledge funding by the Deutsche Forschungsgemeinschaft (DFG) in the frame of the Schwerpunktprogramm (SPP) 1840, Quantum Dynamics in Tailored Intense Fields. We thank L. Yue and W. Pfeiffer for fruitful discussions. We thank $\mathrm{T}$. Weber and F. Ronneberger for technical assistance.

*Corresponding author. philipp.wustelt@uni-jena.de

Corresponding author. gerhard.paulus@uni-jena.de
${ }^{\ddagger}$ Present address: Benedictine College, Atchison, Kansas 66002, USA.

[1] H. H. Nielsen, Rev. Mod. Phys. 23, 90 (1951).

[2] C. Aieta, M. Micciarelli, G. Bertaina, and M. Ceotto, Nat. Commun. 11, 4348 (2020).

[3] P. Wustelt, M. Kübel, G. G. Paulus, and A. M. Sayler, Adv. At. Mol. Opt. Phys. 69, 67 (2020).

[4] E. T. Nibbering, H. Fidder, and E. Pines, Annu. Rev. Phys. Chem. 56, 337 (2005).

[5] J. J. Tanner and M. M. Maricq, Phys. Rev. A 40, 4054 (1989).

[6] A. Guldberg and G. D. Billing, Chem. Phys. Lett. 186, 229 (1991).

[7] A. Giusti-Suzor, X. He, O. Atabek, and F. H. Mies, Phys. Rev. Lett. 64, 515 (1990).

[8] A. S. Alnaser, M. Kübel, R. Siemering, B. Bergues, N. G. Kling, K. J. Betsch, Y. Deng, J. Schmidt, Z. A. Alahmed, A. M. Azzeer, J. Ullrich, I. Ben-Itzhak, R. Moshammer, U. Kleineberg, F. Krausz, R. De Vivie-Riedle, and M. F. Kling, Nat. Commun. 5, 3800 (2014).

[9] Y. Gu and J.-M. Yuan, Phys. Rev. A 36, 3788 (1987).

[10] M.E. Goggin and P. W. Milonni, Phys. Rev. A 38, 5174 (1988).

[11] P. Tran, Phys. Rev. A 59, 1444 (1999).

[12] L. Windhorn, T. Witte, J. S. Yeston, D. Proch, M. Motzkus, K. L. Kompa, and W. Fuß, Chem. Phys. Lett. 357, 85 (2002).

[13] D. Brinks, F. D. Stefani, F. Kulzer, R. Hildner, T. H. Taminiau, Y. Avlasevich, K. Müllen, and N. F. Van Hulst, Nature (London) 465, 905 (2010).

[14] T. Stensitzki, Y. Yang, V. Kozich, A. A. Ahmed, F. Kössl, O. Kühn, and K. Heyne, Nat. Chem. 10, 126 (2018).

[15] J. G. Kim, S. Nozawa, H. Kim, E. H. Choi, T. Sato, T. W. Kim, K. H. Kim, H. Ki, J. Kim, M. Choi, Y. Lee, J. Heo, K. Y. Oang, K. Ichiyanagi, R. Fukaya, J. H. Lee, J. Park, I. Eom, S. H. Chun, S. Kim, M. Kim, T. Katayama, T. Togashi, S. Owada, M. Yabashi, S. J. Lee, S. Lee, C. W. Ahn, D. S. Ahn, J. Moon, S. Choi, J. Kim, T. Joo, J. Kim, S. ichi Adachi, and H. Ihee, Nature (London) 582, 520 (2020).

[16] A. H. Zewail, J. Phys. Chem. A 104, 5660 (2000).

[17] See Supplemental Material at http://link.aps.org/ supplemental/10.1103/PhysRevLett.127.043202 for details of the experiments, simulations and the transition dipole elements for vibrational excitations in $\mathrm{HeH}^{+}$.

[18] N. Bloembergen and A. H. Zewail, J. Phys. Chem. 88, 5459 (1984).

[19] K. I. Dimitriou, V. Constantoudis, T. Mercouris, Y. Komninos, and C. A. Nicolaides, Phys. Rev. A 76, 033406 (2007).

[20] S. Chelkowski, A. D. Bandrauk, and P. B. Corkum, Phys. Rev. Lett. 65, 2355 (1990).

[21] S. Gräfe, P. Marquetand, V. Engel, N. E. Henriksen, and K. B. Møller, Chem. Phys. Lett. 398, 180 (2004).

[22] S. Gräfe, C. Meier, and V. Engel, J. Chem. Phys. 122, 184103 (2005).

[23] D. J. Maas, D. I. Duncan, R. B. Vrijen, W. J. Van Der Zande, and L. D. Noordam, Chem. Phys. Lett. 290, 75 (1998).

[24] C. Ventalon, J. M. Fraser, M. H. Vos, A. Alexandrou, J. L. Martin, and M. Joffre, Proc. Natl. Acad. Sci. U.S.A. 101, 13216 (2004).

[25] V. S. Prabhudesai, U. Lev, A. Natan, B. D. Bruner, A. Diner, O. Heber, D. Strasser, D. Schwalm, I. Ben-Itzhak, J. J. Hua, 
B. D. Esry, Y. Silberberg, and D. Zajfman, Phys. Rev. A 81, 023401 (2010).

[26] T. Witte, T. Hornung, L. Windhorn, D. Proch, R. De VivieRiedle, M. Motzkus, and K. L. Kompa, J. Chem. Phys. 118, 2021 (2003).

[27] D. B. Strasfeld, S.-H. Shim, and M. T. Zanni, Phys. Rev. Lett. 99, 038102 (2007).

[28] J. Loreau, J. Liévin, P. Palmeri, P. Quinet, and N. Vaeck, J. Phys. B 43, 065101 (2010).

[29] D. Ursrey, F. Anis, and B. D. Esry, Phys. Rev. A 85, 023429 (2012).

[30] P. Wustelt, F. Oppermann, L. Yue, M. Möller, T. Stöhlker, M. Lein, S. Gräfe, G. G. Paulus, and A. M. Sayler, Phys. Rev. Lett. 121, 073203 (2018).

[31] F. Oppermann, P. Wustelt, T. Florin, S. Mhatre, S. Gräfe, G. G. Paulus, and M. Lein, J. Phys. B 53, 174001 (2020).
[32] T. R. Hogness and E. G. Lunn, Phys. Rev. 26, 44 (1925).

[33] J. Loreau, J. Lecointre, X. Urbain, and N. Vaeck, Phys. Rev. A 84, 053412 (2011).

[34] W. Ketterle, H. Figger, and H. Walther, Phys. Rev. Lett. 55, 2941 (1985).

[35] P. F. Endres and D. J. Wilson, J. Chem. Phys. 46, 425 (1967).

[36] E. F. de Lima and J. E. M. Hornos, J. Chem. Phys. 125, 164110 (2006).

[37] N. V. Vitanov, T. Halfmann, B. W. Shore, and K. Bergmann, Annu. Rev. Phys. Chem. 52, 763 (2001).

[38] T. Endo, S. P. Neville, V. Wanie, S. Beaulieu, C. Qu, J. Deschamps, P. Lassonde, B. E. Schmidt, H. Fujise, M. Fushitani, A. Hishikawa, P. L. Houston, J. M. Bowman, M. S. Schuurman, F. Légaré, and H. Ibrahim, Science 370, 1072 (2020). 